\title{
Hacia la búsqueda de efectividad en promoción de la salud en América Latina
}

\author{
Searching evidence of health promotion \\ effectiveness in Latin America
}

Ligia de Salazar 1

Jenny Andrea Vélez 2

\footnotetext{
1 Proyecto Regional Latinoamericano de Evidencias de Efectividad en Promoción de la Salud. Directora Centro para el Desarrollo y Evaluación de Políticas y Tecnología en Salud Pública, CEDETES. Universidad del Valle, Calle 4B no 36-00 San Fernando, Piso 1AA 20637 Cali, Colombia. cedetes@cedetes.og

2 Proyecto Regional

Latinoamericano de Evidencias de Efectividad en Promoción de la Salud.
}

Abstract Health promotion in the Latin America region has been expressed not only as a goal but as a political will of governments, health institutions and educative centers. The implementation of its principles and components is in itself a challenge in a region where health determinants and therefore the conditions to be healthy are far from the expected, demanding intersectoral actions and sometimes complex interventions that goes beyond the health sector and individual actions. It has been recognized the need to evaluate its achievements and effects in both, people capacity to deal with health problems and its determinants and changes in health status and conditions to be healthy. This task constitutes not only an ethical imperative but a methodological and political challenge which has been faced through the project Evidences of Effectiveness in Health Promotion in Latin America as an initiative of the IUHPE. Awareness, training, evaluation research, information dissemination and advocacy, are the main strategies and activities of the project, in order to strength the regional capacity and infrastructure to develop, publish and use evaluations results to influence decisions regarding health promotion.

Key words Effectiveness, Evidences, Evaluation, Regional Project, Latin America, Health Promotion
Resumen La promoción de la salud en América Latina ha sido expresada no solo como una meta sino como una voluntad politica de gobiernos, instituciones de salud y centros educativos. La implementación de sus principios y componentes se convierte en un reto, en una región donde las condiciones para ser saludable y las capacidades de controlar determinantes de salud están lejos de las expectativas. Desde esta perspectiva ha sido ampliamente reconocida la necesidad de evaluar los logros y efectos de la promoción de la salud, en términos de las capacidades para lograr cambios en el estado de salud y sus determinantes. Esta tarea se está afrontado a través del proyecto de Evidencias de Efectividad en Promoción de Salud en América Latina, promovido por la UIPES. Concientización, formación, investigación evaluativa, diseminación de información y abogacía son las estrategias principales y actividades del proyecto, con el fin de fortalecer la capacidad regional para desarrollar, publicar y usar resultados de las evaluaciones para influir en decisiones relacionadas con promoción de salud.

Palabras claves Efectividad, Evidencias, Evaluación, Proyecto regional, Latinoamérica, Promoción de la Salud 


\section{Introducción}

Casi dos décadas han transcurrido desde la promulgación de la Carta de Ottawa (WHO, 1986) en las cuales múltiples iniciativas se han generado en América Latina para operar la promoción de la salud. Sin embargo, en estos cerca de 20 años, siguen sin responder preguntas en torno a los alcances de la promoción de la salud relacionados con el éxito de las políticas e intervenciones, con los factores que han determinado su éxito o fracaso y los costos económicos, sociales, políticos y culturales que conlleva alcanzarlos (De Salazar, Vélez y Ortiz, 2003).

La necesidad de disponer de información sobre la efectividad y rentabilidad social de intervenciones en promoción de la salud es en la actualidad ampliamente reconocida, y cada día crecen las demandas por decisiones informadas como producto de estudios de efectividad, relevancia y utilidad de las políticas y programas (UIPES, 1999).

Ha habido un especial énfasis sobre la urgencia de trabajar en el tema afrontando su complejidad. Recientes trabajos realizados en el contexto europeo han hecho exigentes búsquedas y análisis en este sentido, y han logrado construir un panorama de la actividad evaluativa en promoción de la salud, identificando y confirmando una gama de temáticas y acciones, así como la multiplicidad de conceptos que le subyacen (WHO, 2001).

Pero si bien el tema ofrece muchas oportunidades para responder a limitantes del pasado y del presente, también enfrenta muchos retos que América Latina tiene que superar. Entre los más sobresalientes están la carencia de un marco conceptual sobre el significado y alcance de la aplicación de evidencia en la formulación de recomendaciones y políticas en promoción de la salud; la limitada capacidad técnica para realizar estudios sobre evidencias en promoción de la salud; la limitada importancia sobre el tema e insuficiente asignación de recursos para investigación en el campo; cultura para la toma de decisiones que no toma en cuenta la evidencia; desconocimiento de la naturaleza y racionalidad de las decisiones, y las percepciones de la comunidad sobre sus necesidades y expectativas (De Salazar, 2002).

De acuerdo con Macdonald, Veen y Tones (1996) la promoción de la salud, al igual que otras estrategias dirigidas a mejorar la salud, está obligada a probar la efectividad de sus in- tervenciones, de lo contrario estaría expuesta a la crítica científica y al escepticismo político. El dilema que emerge de este análisis es que las formas de acción más poderosas en promoción de la salud son aquellas que parecen arrojar resultados a largo plazo y las cuales son difíciles de predecir, controlar y medir por medios convencionales.

La evaluación de efectividad de intervenciones de naturaleza participativa, como las que se desarrollan en promoción de la salud, deberá considerar, entre otros aspectos, la naturaleza cambiante de los procesos de promoción de la salud; el carácter multidimensional de su acción, en donde se presenta, en forma concomitante, diversas intervenciones con objetivos específicos, aunque complementarios; su orientación a grupos poblacionales, generalmente con una alta movilidad; sus efectos a largo plazo, muchas veces difíciles de medir y costear; y de beneficios frecuentemente intangibles para los tomadores de decisión.

$\mathrm{Al}$ concebir la promoción de la salud como un proceso político de construcción de capacidad para proteger y mantener la salud y controlar sus determinantes (Kickbusch 1996), se entenderá que la evaluación debe centrarse tanto en la evolución de este proceso como en sus efectos en la salud y bienestar de la población (De Salazar, 2003).

Por tanto, la recolección de evidencias para valorar la promoción de la salud, sigue siendo una tarea desafiante. Se necesita una visión amplia de evidencia que abarque la complejidad inherente de la promoción de la salud como un campo. McQueen (2000) afirma que es un reto para la promoción de la salud convencer a sus entusiastas y detractores que no hay ninguna respuesta fácil a los complejos fenómenos humanos.

Muchas experiencias alrededor de los anteriores aspectos han sido documentadas y publicadas, particularmente en Europa y Norteamérica, pero poco se conoce sobre los esfuerzos adelantados en la región latinoamericana y la efectividad de los mismos.

Como una estrategia para encarar los desafíos y las limitaciones mencionadas, la Unión Internacional de Promoción y Educación para la Salud, UIPES, puso en marcha en el 2002 el Proyecto Regional de Evidencias de Efectividad en Promoción de la Salud en América Latina, en el marco del Programa Global de Efectividad en Promoción de la Salud, GPHPE, que había iniciado desde 1999 con el apoyo de los 
Centros para la Prevención y Control de Enfermedades, CDC, de Atlanta, USA; la Organización Mundial de la Salud / Organización Panamericana de la salud, OMS/OPS; y un sinnúmero de instituciones y organizaciones de todos los continentes, interesadas en promover la temática.

La iniciativa regional se concretó durante la III Conferencia Latinoamericana de Promoción y Educación para la Salud, organizada por la UIPES en noviembre de 2002 en São Paulo, Brasil, y además de las instituciones promotoras del Programa Global, contó con el apoyo de la Oficina Regional Latinoamericana de la UIPES, ORLA; el Consorcio Interamericano de Centros de Formación en Promoción de la Salud; La Asociación Brasilera de Salud Colectiva, Abrasco; el Centro para el Desarrollo y Evaluación de Tecnología en Salud Pública, CEDETES, de la Universidad del Valle; los Ministerios de Salud de varios países, diversas universidades de la Región, y otras organizaciones e instituciones que se fueron articulando a medida que se difundió el proyecto.

Este nuevo proyecto se propondría crear capacidad regional para desarrollar, publicar y usar evaluaciones para influenciar decisiones relacionadas con la Promoción de la Salud, a través de incentivar compromisos, voluntades y estrategias para la promoción y el fortalecimiento de la actividad evaluativa en Promoción de la Salud en Latinoamérica; identificar y sintetizar metodologías de evaluación; promover cultura de la evaluación y su uso en decisiones; $\mathrm{y}$ fortalecer la formación y entrenamiento regional en torno al tema.

\section{Métodos}

Concientización y motivación, capacitación y entrenamiento, investigación evaluativa, diseminación de información y abogacía son las principales estrategias y actividades del Proyecto Regional.

Con esta perspectiva, la agenda durante el primer año incluyó la creación de una red regional de instituciones, organizaciones y personas interesadas en la temática, la cual se logró empleando estrategias de comunicación, intercambio de información y el uso de herramientas de Internet para su convocatoria y movilización.

Esta agenda también contempló actividades de reflexión sobre la definición operacional de la promoción de salud en el contexto latinoamericano; un estudio exploratorio para conocer la situación de la promoción de la salud en América Latina; y una base de datos de experiencias relacionadas con efectividad de intervenciones de promoción de salud.

El exploratorio, que identificó 149 estudios relacionados con Promoción de la Salud en América Latina, se basó en dos métodos para la recolección de la información: la revisión de la literatura indexada en las bases de datos Medline y Lilacs, y un cuestionario para el sondeo de experiencias "Aprendiendo a Re-conocernos", que se distribuyó entre la red de participantes del proyecto.

El procesamiento y análisis de la información del exploratorio se realizó mediante matrices descriptivas y temáticas para categorizar y clasificar los estudios, diseñadas especialmente para esta tarea. Concomitante con el análisis temático, se realizó análisis por tipos de estudio y diseños, número de estudios, años de publicación, procedencia geográfica y peso de la producción de la literatura indexada, fuentes e idiomas.

En el análisis se tomó en cuenta los componentes de Promoción de la salud definidos en la Carta de Ottawa para responder tres preguntas: ¿Hacia dónde se orienta la promoción de la salud en América Latina?, ¿Evaluamos y qué evaluamos en promoción de la salud? y ¿Dónde podemos actuar considerando el panorama y las expectativas?

Responder eficazmente a los vacíos y desafíos hallados en el estudio exploratorio y a las limitaciones anteriormente expuestas fue el propósito de la agenda del proyecto para el segundo año.

Esta ha implicado el desarrollo metodológico de diseños de evaluación apropiados a las necesidades regionales; estrategias para aumentar la viabilidad e impacto de la evaluación de efectividad, como el diseño de metodologías para sistematizar intervenciones de promoción de salud, entre ellas la Evaluación de Proceso y la Evaluación Participativa que se están llevando a cabo con el liderazgo de la OPS; y metodologías para articular la vigilancia de factores de riesgo a iniciativas de evaluación en la Región.

Para concretar estas estrategias se diseñó y puso en marcha un programa de entrenamiento para América Latina, en la metodología de Evaluación Rápida, que combina actividades de aprendizaje presenciales y a distancia, con el fin de proveer una tecnología de evaluación de 
fácil adopción, en menor tiempo y con menos recursos que las tradicionales. En este programa han participado 13 representantes de seis países, la mayoría directivos de agencias gubernamentales y académicos.

La agenda del tercer año del proyecto se orientará a incentivar la capacitación regional como estrategia central para construir capacidad, y en la realización de cuatro actividades fundamentales:

1) aportes conceptuales y metodológicos en torno a la evaluación en promoción de la salud y la construcción de evidencias de su efectividad;

2) la realización de estudios de casos en los países, que permita identificar y sintetizar evidencias o "señales" de efectividad en promoción de la salud;

3) la publicación y diseminación de las experiencias;

4) abogacía para la realización de estudios colaborativos que fortalezcan los resultados obtenidos.

\section{Resultados}

Las actividades del Proyecto Regional han mostrado, entre otros, una alta motivación en la región latinoamericana para participar en iniciativas de promoción de salud y de evaluación, pero también acciones limitadas alrededor de la provisión de servicios de salud y de intervenciones en determinantes de salud.

El proyecto se enfrenta a grandes limitaciones regionales en capacidad técnica y de recursos para dirigir estudios de evaluación, en habilidades para escribir artículos científicos, publicar y producir informes según la necesidad de tomadores de decisión, comunidades y otros interesados potenciales de la evaluación, y en promover el uso de la evaluación en políticas y decisiones relacionadas con promoción de la salud.

Sin embargo, en dos años de operación, el proyecto ha logrado promover la temática, incentivar la discusión y movilizar el interés de más de 200 organizaciones, instituciones y personas interesadas en la temática en América Latina.

El Proyecto Regional ha provisto un panorama de la situación de la promoción de la salud y de las iniciativas para evaluarla en América Latina, que está orientando prioridades para la agenda de trabajo del mismo proyecto y de otras iniciativas con propósitos similares o involucradas en el campo.

Se han generado insumos para la construcción de un marco conceptual apropiado a las condiciones, prácticas y experiencias de la promoción de la salud en los países de la región, así como espacios para conocer y re-conocer las experiencias latinoamericanas, en el propósito de cerrar brechas y vacíos de información, y facilitar las condiciones para articular esfuerzos.

Insumos para incrementar las publicaciones de la región en torno a la promoción de la salud y las evidencias de su efectividad, así como facilitar condiciones para que la región tenga una mayor y más cualificada participación en la agenda de las principales fuentes de información en torno al tema, se constituyen también en resultados del proyecto.

El Programa de Entrenamiento en Evaluación ha permitido, entre otros resultados, la generación de condiciones para impulsar la evaluación en los países. Producto del programa actualmente se desarrollan ocho procesos evaluativos en Latinoamérica, orientados a buscar "señales" de efectividad de las intervenciones de promoción de la salud. Se espera que, como mínimo, igual número de publicaciones en torno al tema sean generadas desde la región.

La información que el proyecto ha logrado recopilar y sintetizar a través de sus diferentes estrategias, será también el punto de partida para fortalecer el trabajo en red y desarrollar la propuesta de creación de un Observatorio Latinoamericano en Promoción de la Salud.

\section{Discusión}

Las actividades del Proyecto Regional han reiterado que la evaluación en promoción de la salud es una temática de gran interés y motivación, pero de poca acción en América Latina, por los limitados conocimientos metodológicos y recursos para hacerlo.

Las actividades alrededor del Proyecto Regional ha permitido establecer algunos acuerdos y desacuerdos sobre la naturaleza y alcance de la promoción de la salud en América Latina. En relación con evaluación de efectividad, ha brindado pistas sobre el interés y capacidad de los países latinoamericanos en torno a la evaluación y diseminación de información, al igual que posibles aportes de las evaluaciones al fortalecimiento conceptual y práctico de la promoción de la salud. 
Sin embargo, la omisión de temas inherentes a la concepción teórica y práctica de promoción de la salud, encontrada en el estudio exploratorio y en las prioridades identificadas, plantea la hipótesis de que en América Latina se carece de una definición operativa consensuada sobre este concepto y que no se han establecido relaciones y diferencias entre atención primaria, promoción de la salud y la definición, ya revaluada, de salud pública.

Las experiencias sintetizadas a través del proyecto muestran que en la región no se cubren aspectos de promoción de la salud que signifiquen cambios estructurales en el marco de los determinantes de la salud, sino más bien intervenciones puntuales de prevención y control orientadas a mejorar el acceso y calidad a los servicios.

La relativa baja importancia dada a los temas de empoderamiento y construcción de capacidad comunitaria para el control de determinantes de salud, observada en la revisión de literatura y en el sondeo de experiencias, se constituyen en una preocupante falencia, considerando que estos dos temas son claves para lograr la equidad y capacidad para intervenir las situaciones coyunturales que afectan la salud, calidad de vida y justicia social y, por tanto, en puntos clave que debe abordar el proyecto.

Considerando que la falta de relevancia y coherencia de las evaluaciones con las necesidades e intereses de los tomadores de decisión en los tiempos políticos ha sido ampliamente cuestionada (Crew y Young, 2002) estos hallazgos llevan a reflexionar sobre la contribución que los resultados de la promoción de la salud están haciendo a la formulación de políticas saludables en los países de la región. Cabe preguntarse si ¿Es ésta la información que los decisores y políticos necesitan y quieren recibir? ¿Se pueden lograr los impactos deseados? ¿Cuál sería el alcance de las decisiones que pueden tomarse con estos resultados? ¿Qué estrategias debe implementar el proyecto para hacer frente a esta situación? ¿Estamos en capacidad de actuar teniendo en cuenta la necesidad de producir evidencias sobre efectividad de la promoción de la salud y ante el contexto descrito?

Responder afirmativamente no es tarea fácil pero sí posible, teniendo en cuenta también las fortalezas que existen en la región y la voluntad y compromiso real de actores claves para el desarrollo de la promoción de la salud en América Latina.

En ese sentido, la iniciativa latinoamericana de evidencias de efectividad en promoción de la salud, promovida por la UIPES, debe aprovechar dichas fortalezas y orientar su agenda de trabajo hacia la creación y fortalecimiento de alianzas con los actores interesados, a fin de conjugar esfuerzos y potenciar recursos para la creación de un proyecto latinoamericano que trascienda el diagnóstico de la situación, para cubrir los puntos neurálgicos que él mismo está señalando, los cuales no han sido desconocidos para la gran mayoría de los estudiosos del tema.

Se hace necesario continuar abierto el debate sobre evidencias en salud pública y promoción de la salud con audiencias que estén en la arena política; estrechar los consensos sobre lo que constituye evidencia, efectividad y los indicadores para evaluarla en el contexto de las iniciativas latinoamericanas; iniciar el debate en torno a la sostenibilidad y a la necesidad de que el Proyecto Regional establezca estrategias que aglutinen, cohesionen y promuevan la acción evaluativa en forma permanente, pues se trata de una iniciativa que se reitera importante pero que no podrá sobrevivir solo de voluntades.

Alrededor del proyecto también se han identificado algunos retos que van surgiendo y deben responderse. El primero se relaciona con la metodología de evaluación y con la aplicación de los resultados, pues ha conciencia de que conocimiento no necesariamente significa práctica. El cubrir la brecha entre conocimiento, formulación de políticas públicas y prácticas para promover la salud y bienestar, implica acciones que rebasan los diseños metodológicos. Las actividades para cumplir con este objetivo implican, entre otras, definir audiencias, canalizar la información según sus intereses, propiciar encuentros para socializar el conocimiento, llegar a consensos y crear opinión pública.

El segundo reto es el fortalecimiento de la propuesta metodológica de evaluación rápida, como el más apropiado de acuerdo con las condiciones de la región y como el primer eslabón en la cadena de la búsqueda de efectividad en promoción de la salud en América Latina.

El tercer reto se relaciona con la creación de capacidad local que haga viable la evaluación en los países. Lo anterior demanda la creación de cultura política y evaluativa que rescate la importancia y el papel de la evaluación en las decisiones de política en promoción de la salud. Igualmente, demanda eficientes sistemas de información y comunicación que hagan de la evaluación un ejercicio menos complejo, más relevante, más participativo y a un costo razonable. 
Finalmente, el cuarto reto es la formación de funcionarios responsables de la evaluación de iniciativas en promoción de la salud, así como de líderes comunitarios, estudiantes y profesionales de la salud. Esta actividad facilita el que la evaluación sea un producto de sucesivas aproximaciones. Por ello, nuestro reto central es hacer de la evaluación un instrumento técnico y político para el cambio social en América Latina.

\section{Contribución de las autoras}

L Salazar participó en la concepción general del artículo, en la revisión crítica de los borradores y versión final, contribuyó en la introducción y marco teórico, resultados y definición de retos y perspectivas. JAV Vidal participó en la redacción de las versiones preliminares y finales del artículo; revisión bibliográfica y contribuyó en la sección de "Estrategias y actividades e identificación de resultados".

\section{Referencias bibliográficas}

Crew E \& Young J 2002. Bridging research and policy: context, evidence and links. (Working Paper 173). Results of ODI research presented in preliminary form for discussion and critical comment. Global Development Network. Overseas Development Institute, UK.

De Salazar L 2002. Municipios y comunidades saludables. El reto de la evaluación. Centro para el Desarrollo y Evaluación de Políticas y Tecnología en Salud Pública, CEDETES. Universidad del Valle. Santiago de Cali, Colombia.

De Salazar L, Vélez JA \& Ortiz Y 2003. Revisión de literatura de evidencias de efectividad en promoción de la salud en América Latina. Proyecto Regional Latinoamericano de Evidencias de Efectividad en Promoción de la Salud. Unión Internacional de Promoción de la Salud y Educación para la Salud, UIPES. Santiago de Cali, Colombia.

Kickbusch I 1996. Promoción de la salud: una perspectiva mundial, pp. 6-14. In Promoción de la salud: una antología. Organización Panamericana de la Salud. Publicación Científica No 557.

McDonald G, Veen C \& Tones K 1996. Evidence for success in health promotion: suggestions for improvement. Health Education Research 11(3):367-376.

McQueen D 2000. V Conferencia Mundial de Promoción de la Salud. Informe Técnico 1. Bases Científicas para la Promoción de la Salud. Ciudad de México, 5 al 9 de junio de 2000.

[UIPES] Unión Internacional de Promoción y Educación para la Salud 1999. La Evidencia de la Eficacia de la Promoción de la Salud. Configurando la Salud Pública en una Nueva Europa. Informe de la UIPES para la Comisión Europea. Evidence Book. Madrid

[WHO] World Health Organization 1986. Carta de Ottawa para la Promoción de la Salud.

[WHO] World Health Organization 2001. Evaluation in Health Promotion. Principles and Perspectives. Editado por Irving Rootman et al. WHO Regional Publications, European Series, No 92.

Artículo presentado en 16/4/2004

Aprobado en 10/5/2004

Versión final presentada en 18/5/2004 